\title{
Optimization of RGD-modified Nano-liposomes Encapsulating Eptifibatide
}

\author{
Hassan Bardania ${ }^{1}$, Seyed Abbas Shojaosadati 2*, Farzad Kobarfard ${ }^{* *}$, Farid Dorkoosh 4 \\ ${ }^{1}$ Department of Nanobiotechnology, Faculty of Biological Sciences, Tarbiat Modares University, Tehran, Iran \\ ${ }^{2}$ Department of Biotechnology Group Chemical Engineering, Tarbiat Modares University, Tehran, Iran \\ ${ }^{3}$ Department of Medical Chemistry, Faculty of Pharmacy, Shahid Beheshti University of Medical Sciences, Tehran, Iran \\ ${ }^{4}$ Department of Pharmaceutics, School of Pharmacy, Tehran University of Medical Sciences, Tehran, Iran \\ *Corresponding author: Seyed Abbas Shojaosadati, Department of Biotechnology Group, Faculty of Chemical Engineering, Tarbiat Modares University, \\ Tehran, Iran. Tel: +98-2182883341, Fax: +98-2182883381, E-mail: shoja_sa@modares.ac.ir \\ ** Co-Corresponding author: Farzad Kobarfard, Department of Medical Chemistry, Faculty of Pharmacy, Shahid Beheshti University of Medical Sciences, \\ Tehran, Iran. Tel: +98-2188200092, E-mail: kobarfard@sbmu.ac.ir
}

Received: November 17, 2015; Revised: January 09, 2016; Accepted: February 14, 2016

Background: Eptifibatide (Integrilin) is an intravenous (IV) peptide drug that selectively inhibits ligand binding to the platelet GP IIb/IIIa receptor. It is an efficient peptide drug, however has a short half-life. Therefore, antithrombotic agents like eptifibatide are required to become improved with a protected and targeted delivery system such as using nano-liposomes to the site of thrombus.

Objectives: The goal in the present report was to optimize encapsulation efficiency of the eptifibatide into Arg-Gly-Asp (RGD)-modified nano-liposomes (RMNL). As well, it was intended to evaluate the effect of sodium lauryl sulfate (SLS) on drug release.

Materials and Methods: The effect of five independent variables including number of freeze/thawing cycles, concentration of eptifibatide, 1,2-distearoyl-sn-glycero-3-phosphocholine (DSPC), cholesterol, and dipalmitoyl-GRGDSPA peptide on drug entrapment efficiency (DEE) was investigated using response surface methodology (RSM). The effect of different concentrations of SLS on encapsulation and drug release from RMNL was also investigated. The size and morphology of RMNL were characterized using transmission electron microscopy (TEM).

Results: The maximum DEE (38\%) was obtained with 7 freeze/thawing cycles, $3.65 \mu \mathrm{moL}$ eptifibatide, $7 \mathrm{mM}$ DSPC, 3 $\mathrm{mM}$ cholesterol, and $1 \mathrm{mM}$ dipalmitoyl- GRGDSPA peptide. SLS has significantly increased the drug release from RMNL, although its effect on encapsulation efficiency was not significant.

Conclusions: The optimization of the formulations for valuable and expensive peptide drugs is essential to have the maximum encapsulation efficiency and the minimum experiments.

Keywords: Eptifibatide; RGD-modified nano-liposomes (RMNL); RMNL encapsulated eptifibatide

\section{Background}

Atherosclerosis is among the major causes of the cardiovascular disease, which results from platelet aggregation, plaque formation, ending to the occlusion of the blood vessels (1). There are chemical, protein, and peptide drugs such as eptifibatide for treating the atherosclerotic plaques efficiently. Eptifibatide (Integrilin) is an intravenous (IV) drug; a cyclic heptapeptide containing 6 amino acids and 1 mercaptopropionyl (des-amino cysteinyl) residue with an interachain disulfide bridge between the cysteine amide and the mercaptopropionyl moieties that selectively inhibits ligand binding to the platelet GP IIb/IIIa receptor and rapidly dissociate from its receptor $(2,3)$. Eptifibatide is an efficient non immunogenic drug, however has a short half-life due to rapid inactivation and elimination by renal filtration, enzymatic degradation, and accumulation in non-targeted tissues (2). Therefore, the efficiency of the antithrombotic agents such as eptifibatide could be improved through protection and targeted delivery using nano-carriers to the site of thrombus (4).

Liposomes are the most commonly used nano-carriers for drug delivery in cardiovascular diseases $(5,6)$. 
Nano-liposomes have nomerous advantages, such as, biocompatibility, low immunogenicity, protection of the cargo against enzymatic degradation, in addition to the easy surface modification for the targeted delivery (7). Activated platelets are one of the most important targets for the targeted thrombolytic therapy. Following to the vascular injury platelet activation leads to the clot formation and restenosis (8).

In several studies, it has been shown that surface modified liposomes by fibrinogen-mimetic RGD motif achieve an affinity toward activated platelets and may be useful for the targeted delivery of thrombolytic agents (8-12). Vaidya et al. (2011) have developed RGD modified liposome for targeted delivery of streptokinase toward activated platelets (9).

\section{Objectives}

The main objective of this study was to develop eptifibatide encapsulation in the RGD-modified nano-liposomes (RMNL) by optimizing drug entrapment efficiency (DEE) into RMNL utilizing response surface methodology (RSM). Central composite design (CCD) was developed using Design Expert Software (DX-7, State-Ease Inc., Version 7.0.0) to evaluate the effect of the number of freeze/thaw cycles, and concentration of eptifibatide, 1, 2distearoyl-sn-glycero-3-phosphocholine (DSPC), cholesterol, and dipamitoyl-GRGDSPA peptide on the DEE of eptifibatide into RMNL. The size and morphology of the prepared liposomes were characterized utilizing transmission electron microscopy (TEM). Drug release was investigated using dialysis in both simulated body fluids (SBF) and SBF containing $0.5 \%$ sodium lauryl sulfate (SLS). SLS was incorporated in RMNL and its influence on DEE and drug release was evaluated.

\section{Materials and Methods}

\subsection{Materials}

Dimethylformamide, N-methyl pyrolidone, and other solvents were purchased from Merck (Germany). 9-fluorenylmethyloxycarbonyl (Fmoc)-Lys (Fmoc)$\mathrm{OH}$ was obtained from AAPPTEC (USA). Rink Amide MBHA resin and other protected amino acids were obtained from GLS China. 1, 2-distearoyl-sn-glycero3-phosphocholine (DSPC) was purchased from Avanti Polar Lipids (USA), and cholesterol from Sigma Aldrich (USA). Eptifibatide was provided by the School of Pharmacy, Shahid Beheshti University of Medical Science (Tehran, Iran). All materials were used without any further purification.

\subsection{Dipalimitoyl-Peptide Synthesis}

Dipalmitoyl-GRGDSPA peptide conjugate was constructed from a GRGDSPA peptide head group, a KGG spacer and 2 palmitic acid (pal) anchors. This construct was synthesized manually by solid-phase peptide synthesis on Rink Amide MBHA resin using standard 9-fluoromethoxycarbonyl (Fmoc) chemistry (13). The dipalmitoyl-GRGDSPA peptide conjugate was integrated into liposomes by the pal anchors which were intercalated within the lipid bilayer. The prepared peptide was analyzed by high performance liquid chromatography (HPLC) and LC-MS spectrometry (Agilent Technology Series).

\subsection{Liposomes Preparation and Drug Incorporation}

RMNL were prepared by thin film evaporation method $(14,15)$. Briefly, the required amounts of DSPC, cholesterol and dipalmitoyl-GRGDSPA peptide were dissolved in $2 \mathrm{~mL}$ chloroform and evaporated using round-bottomed flask under low vacuum in order to form a thin lipid film. The lipid film was hydrated at $65^{\circ} \mathrm{C}$ in $50 \mathrm{mM}$ Tris buffer at $\mathrm{pH} 7.4$ for approximately $2 \mathrm{~h}$. Eptifibatide solution was prepared in $50 \mathrm{mM}$ Tris buffer, $\mathrm{pH} 7.4$ at the desired amounts. The solution was mixed with the prepared drug free RMNL using different concentration of phospholipids and performing freeze/thawing cycles: the mixture was frozen in the liquid nitrogen $\left(-196^{\circ} \mathrm{C}\right)$ for $5 \mathrm{~min}$ and thawed in water bath at $65^{\circ} \mathrm{C}$ and shaking at 150 rpm for $5 \mathrm{~min}$. Different cycles were performed to promote the encapsulation of eptifibatide into liposomes. After optimization of the eptifibatide encapsulation, the effect of sodium lauryl sulfate (SLS) was evaluated as a surfactant on drug encapsulation and release. Different concentration of SLS solution in methanol $(0.25,0.5,1$ and $2 \%$ mol of DSPC) was added to phospholipid solution (DSPC and cholesterol and dipalmitoyl-GRGDSPA peptide dissolved in chloroform) and used for formulation of the liposomes.

\subsection{Drug Entrapment Efficiency (DEE) Optimization}

A statistical experimental design based on RSM was used to obtain optimal conditions for DEE. Based on the preliminary experiments and literature survey, five independent variables (the number of freeze/thawing cycles, in addition to concentration of the following components: eptifibatide, DSPC, cholesterol, and dipalmitoyl- GRGDSPA peptide) were selected. CCD was used with the five- variables at five-levels applying $1 / 2$ fraction factorial and six center points resulting in 32 
Bardania H. et al.

\begin{tabular}{|c|c|c|c|c|c|c|}
\hline Run & $\begin{array}{l}\text { The number of } \\
\text { freeze/thawing } \\
\text { cycles }\end{array}$ & $\begin{array}{l}\text { Eptifibatide } \\
(\mu \mathrm{mol})\end{array}$ & DSPC (mM) & $\begin{array}{l}\text { Cholesterol } \\
\text { (mM) }\end{array}$ & $\begin{array}{c}\text { Dipalmitoyl-GRGDSPA } \\
\text { peptide (mM) }\end{array}$ & DEE (\%) \\
\hline 1 & 3 & 1.65 & 6.25 & 3.625 & 0.75 & 0.68 \\
\hline 2 & 6 & 2.7 & 7 & 2.75 & 1.5 & 3.31 \\
\hline 3 & 3 & 1.65 & 6.25 & 1.875 & 1.25 & 8.62 \\
\hline 4 & 8 & 1.65 & 6.25 & 1.875 & 0.75 & 11.16 \\
\hline 5 & 8 & 1.65 & 7.75 & 1.875 & 1.25 & 16.29 \\
\hline 6 & 3 & 1.65 & 7.75 & 3.625 & 1.25 & 20.25 \\
\hline 7 & 6 & 2.7 & 7 & 2.75 & 0.5 & 3.74 \\
\hline 8 & 8 & 3.75 & 7.75 & 1.875 & 0.75 & 24.39 \\
\hline 9 & 3 & 3.75 & 7.75 & 1.875 & 1.25 & 25.85 \\
\hline 10 & 6 & 0.6 & 7 & 2.75 & 1 & 27.28 \\
\hline 11 & 8 & 1.65 & 7.75 & 3.625 & 0.75 & 27.52 \\
\hline 12 & 6 & 2.7 & 5.5 & 2.75 & 1 & 28.14 \\
\hline 13 & 6 & 2.7 & 7 & 1 & 1 & 29.99 \\
\hline 14 & 6 & 2.7 & 7 & 2.75 & 1 & 27.28 \\
\hline 15 & 8 & 3.75 & 6.25 & 1.875 & 1.25 & 32.75 \\
\hline 16 & 6 & 2.7 & 7 & 2.75 & 1 & 35.74 \\
\hline 17 & 1 & 2.7 & 7 & 2.75 & 1 & 33.63 \\
\hline 18 & 8 & 3.75 & 7.75 & 3.625 & 1.25 & 33.65 \\
\hline 19 & 6 & 2.7 & 7 & 4.5 & 1 & 33.68 \\
\hline 20 & 6 & 2.7 & 8.5 & 2.75 & 1 & 34.64 \\
\hline 21 & 6 & 2.7 & 7 & 2.75 & 1 & 36.95 \\
\hline 22 & 3 & 3.75 & 6.25 & 3.625 & 1.25 & 36.23 \\
\hline 23 & 6 & 4.8 & 7 & 2.75 & 1 & 37.79 \\
\hline 24 & 8 & 1.65 & 6.25 & 3.625 & 1.25 & 26.53 \\
\hline 25 & 3 & 1.65 & 7.75 & 1.875 & 0.75 & 28.01 \\
\hline 26 & 6 & 2.7 & 7 & 2.75 & 1 & 30.81 \\
\hline 27 & 8 & 3.75 & 6.25 & 3.625 & 0.75 & 29.74 \\
\hline 28 & 3 & 3.75 & 7.75 & 3.625 & 0.75 & 35.23 \\
\hline 29 & 11 & 2.7 & 7 & 2.75 & 1 & 35.59 \\
\hline 30 & 6 & 2.7 & 7 & 2.75 & 1 & 28.98 \\
\hline 31 & 6 & 2.7 & 7 & 2.75 & 1 & 33.17 \\
\hline 32 & 3 & 3.75 & 6.25 & 1.875 & 0.75 & 5.71 \\
\hline
\end{tabular}

runs (Table 1). The ranges and levels of examined independent variables are listed in (Table 2).

The percentage of DEE as response was calculated using the following equation:

$\operatorname{DEE}(\%)=\mathrm{C}_{\text {initial }}-\mathrm{C}_{\text {free }} / \mathrm{C}_{\text {initial }} \times 100$
Where, $\mathrm{C}_{\text {free }}$ is the non-incorporated drug remained in the hydration medium following to separation of the liposomes by centrifugation at $29000 \times g$ for $30 \mathrm{~min}$. The free drug was measured by using HPLC with UV/visible detector at $220 \mathrm{~nm}$. Briefly, $4.6 \times 15 \mathrm{~nm}$ reverse-phase C18 Zorbax ${ }^{\circledR}$ column (Hewlett-

\begin{tabular}{lcccccc}
\hline Table 2. Experimental variables at different levels used in CCD & & & & \\
\hline Independent Variables & Code & Low & Center & High & Axial $(-\alpha)$ & Axial $(+\alpha)$ \\
\hline The Number of freeze/thawing cycles & X1 & 3 & 5.5 & 8 & 1 & 11 \\
Eptifibatide concentration $(\mu \mathrm{moL})$ & $\mathrm{X} 2$ & 1.65 & 2.7 & 3.75 & 0.6 & 4.8 \\
DSPC concentration $(\mathrm{mM})$ & $\mathrm{X} 3$ & 6.25 & 7 & 7.75 & 5.5 & 8.5 \\
Cholesterol concentration $(\mathrm{mM})$ & $\mathrm{X} 4$ & 1.87 & 2.74 & 3.62 & 1 & 4.5 \\
Dipalmitoyl- GRGDSPA peptide concentration $(\mathrm{mM})$ & $\mathrm{X} 5$ & 0.75 & 1 & 1.25 & 0.5 & 1.5 \\
\hline
\end{tabular}


Packard, Sigma, Germany) was used. The mobile phase was acetonitrile: water with $20: 80(\mathrm{~V} / \mathrm{V})$ ratio containing $0.1 \%$ trifluoroacetic acid. $\mathrm{C}_{\text {initial }}$ is the total drug used for loading drug into RMNL.

The behavior of the system was explained by the quadratic polynomial empirical model (2).

$\mathrm{Y}=\beta_{0}+\sum_{i} \beta_{i} X_{i}+\sum_{i} \beta_{i i} X_{i}^{2}+\sum_{i \neq j} \beta_{i j} X_{i} X_{\mathrm{j}}$

Where $y$ is the expected response; $\beta_{0}, \beta_{\mathrm{i}}, \beta_{\mathrm{ii}}$ and $\beta_{\mathrm{ij}}$ are intercept coefficient, the linear coefficient, the squared coefficient, and the interaction coefficient respectively; $X_{i}$ and $X_{j}$ are coded factors of independent variables $(17,18)$. Here y represents DEE $(\%)$ for the empirical model.

Data were evaluated for Eq.2 by Analysis of Variance (ANOVA) to assess the interaction between the independent variables and the response. The adequacy of the polynomial model was checked by the coefficient of determination $\left(\mathrm{R}^{2}\right)$, and its statistical significance was checked by the F-test (p-value). All the independent variables were kept within the range while the response was maximized.

\subsection{Characterization of Liposomes}

The size and morphology of the prepared RMNL was analyzed by transmission electron microscopy (TEM) (Philips CM30, Netherlands). Sample were mounted on carbon coated copper grids (400 mesh, Agar Scientific, UK) and viewed without any staining. The size distribution and $\zeta$ potential of RMNL was measured at different $\mathrm{pH}(5.5,7.4$ and 8.5) using a Zetasizer Nano ZS instrument (Malvern Instruments Ltd, Malvern, Worcestershire, UK).

\subsection{Evaluation of the Effect of SLS Concentration on DEE and Drug Release}

The influence of SLS on eptifibatide encapsulation and release was evaluated using different concentrations of SLS $\left(0,0.25,0.5,1\right.$ and $2 \%$ moL SLS.mol ${ }^{-1}$ DSPC) for formulation of liposomes. Eptifibatide encapsulation was carried out based on optimum condition obtained from RSM for liposomes containing different concentration of SLS and DEE measured using HPLC analysis.

The in vitro release of eptifibatide from different prepared samples was examined. Briefly, liposomal samples were dispersed in $2 \mathrm{~mL} \mathrm{SBF}$ and transferred into the sealed dialysis tubes (MW cut-off $12000 \mathrm{Da}$ ). The dialysis tubes were submerged in $10 \mathrm{~mL}$ SBF and SBF containing $0.5 \%$ SLS. The tubes were then incu- bated in a thermostatic shaker $\left(37^{\circ} \mathrm{C}, 150 \mathrm{rpm}\right)$ for 24 h. At the pre-determined time intervals, $500 \mu \mathrm{L}$ of SBF containing eptifibatide released from liposomes were used for determination of the released drug concentration by HPLC. The $10 \mathrm{~mL} \mathrm{SBF}$ in the recipient media was taken out and replaced with the fresh SBF at the same time intervals.

\section{Results}

\subsection{Peptide-Dipalmitic Acid Conjugate}

Dipalmitoyl-GRGDSPA peptide was synthesized by Fmoc chemistry used for surface modification of the prepared liposomes through anchoring the two palmitic acid residues to the surface of nanoliposomes. The synthesized di-palmitoyl peptide was characterized by HPLC and LC-MS analysis. Figure 1 demonstrates the structure along with the representative mass spectrum of the peptide-dipalmitic acid conjugate designed for attachment to the GPIIb-IIIa receptor. The purity of dipalmitoyl- GRGDSPA peptide conjugate was confirmed by HPLC analyses.

\subsection{Statistical Analysis}

The optimization of eptifibatide encapsulated liposomes was carried out using CCD (Table 1). The effect of the number of freeze/thaw cycles, and drug, DSPC, cholesterol and dipalmitoyl- GRGDSPA peptide con-
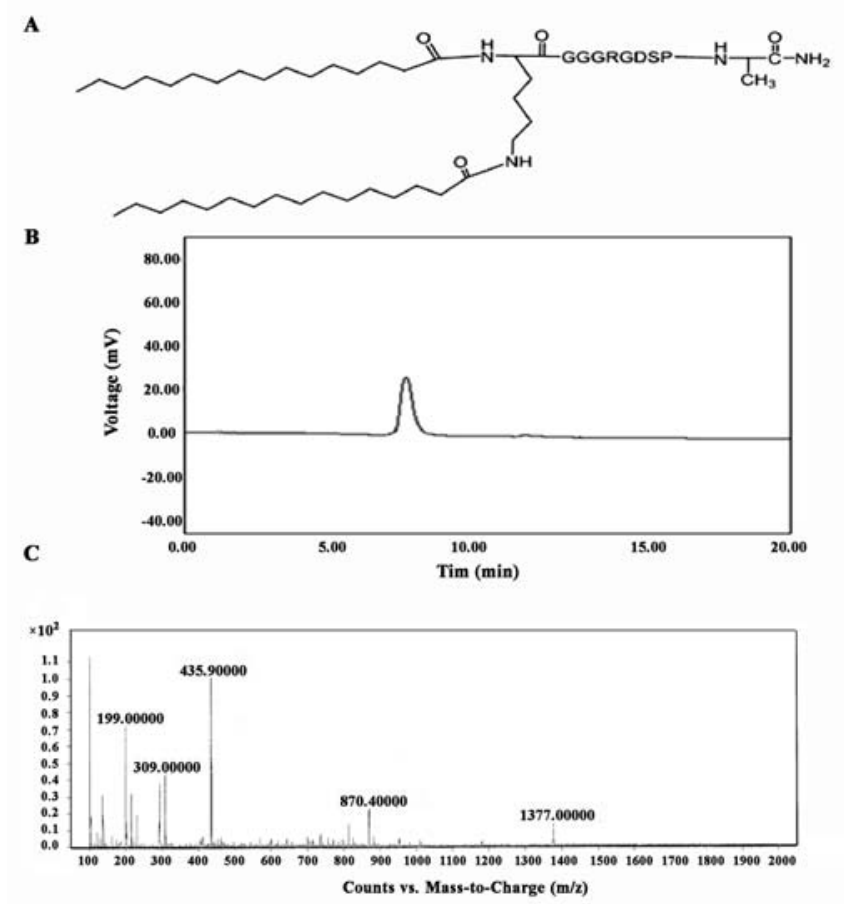

Figure 1. Dipalmitoyl- GRGDSPA peptide conjugate A: the chemical structure B: HPLC chromatogram, and C: mass spectrum 
Bardania H. et al.

Table 3. ANOVA test for Response Surface Reduced Quadratic Model

\begin{tabular}{|c|c|c|c|c|c|c|}
\hline Source & & Sum of Squares & DF & $\begin{array}{c}\text { Mean } \\
\text { Squares }\end{array}$ & F Value & Prob $>$ F \\
\hline Model & & 3468.828507 & 14 & 247.7734648 & 13.90377 & $<0.0001$ \\
\hline A & & 85.72424225 & 1 & 85.72424225 & 4.810404 & 0.0425 \\
\hline B & & 463.6423275 & 1 & 463.6423275 & 26.01723 & $<0.0001$ \\
\hline C & & 220.5161087 & 1 & 220.5161087 & 12.37423 & 0.0026 \\
\hline D & & 173.066687 & 1 & 173.066687 & 9.711614 & 0.0063 \\
\hline$E$ & & 56.67641755 & 1 & 56.67641755 & 3.180389 & 0.0924 \\
\hline$A B$ & & 2.580477562 & 1 & 2.580477562 & 0.144803 & 0.7083 \\
\hline$A C$ & & 198.9317198 & 1 & 198.9317198 & 11.16303 & 0.0039 \\
\hline$A D$ & & 4.66908313 & 1 & 4.66908313 & 0.262005 & 0.6153 \\
\hline$A E$ & & 1.508970496 & 1 & 1.508970496 & 0.084676 & 0.7746 \\
\hline $\mathrm{BC}$ & & 57.73953465 & 1 & 57.73953465 & 3.240046 & 0.0896 \\
\hline BD & & 77.63369473 & 1 & 77.63369473 & 4.356404 & 0.0522 \\
\hline $\mathrm{BE}$ & & 52.89008204 & 1 & 52.89008204 & 2.96792 & 0.1031 \\
\hline CE & & 360.5091007 & 1 & 360.5091007 & 20.22992 & 0.0003 \\
\hline$E^{\wedge} 2$ & & 1712.740061 & 1 & 1712.740061 & 96.11018 & $<0.0001$ \\
\hline Residual & & 302.9500329 & 17 & 17.82059017 & & \\
\hline Lack of Fit & & 230.5030731 & 12 & 19.20858942 & 1.3257 & 0.4010 \\
\hline $\mathrm{R}^{2}$ & 0.9197 & & & & & \\
\hline adj $R^{2}$ & 0.8535 & & & & & \\
\hline
\end{tabular}

Table 4. Point prediction and validation of the DEE at the optimal conditions

\begin{tabular}{lccc}
\hline Confirmation Experiment (\%) & Prediction & 95\% Cl low & 95\% Cl high \\
\hline 37.49 & 38.85 & 35.16 & 41.55 \\
\hline
\end{tabular}

centrations on DEE were evaluated. The result of ANOVA test is presented in (Table 3 ) and $p$-value less than 0.05 was considered as statistically significant at $95 \%$ confidence interval.

\subsection{Fitting Model}

A reduced quadratic model for the defined factors and their interactions are presented in equation 3.

$$
\begin{aligned}
& \mathrm{R} 1-31.40+1.89 \mathrm{~A}+4.40 \mathrm{~B}+3.03 \mathrm{C}+2.69 \mathrm{D}+ \\
& 1.54 \mathrm{E}-0.40 \mathrm{AB}-3.53 \mathrm{AC}+0.54 \mathrm{AD}-0.31 \mathrm{AE}- \\
& 1.90 \mathrm{BC}+2.02 \mathrm{BD}-1.82 \mathrm{BE}-4.75 \mathrm{CE}-7.56 \mathrm{E}^{2}
\end{aligned}
$$

Where A, denotes the freeze/thawing cycle number, B, $\mathrm{C}, \mathrm{D}$, and $\mathrm{E}$ are the concentrations of drug, DSPC, cholesterol and dipalmitoyl-GRGDSPA peptide respectively. The relatively high $\mathrm{R}^{2}$ and adj. $\mathrm{R}^{2}$ values in (Table 3 ) indicate that model is capable of representing the system under the given experimental conditions. Also, clustering of the points around the diagonal line in (Figure 2) has confirmed a satisfactory correlation between the experimental data and the predicted values.

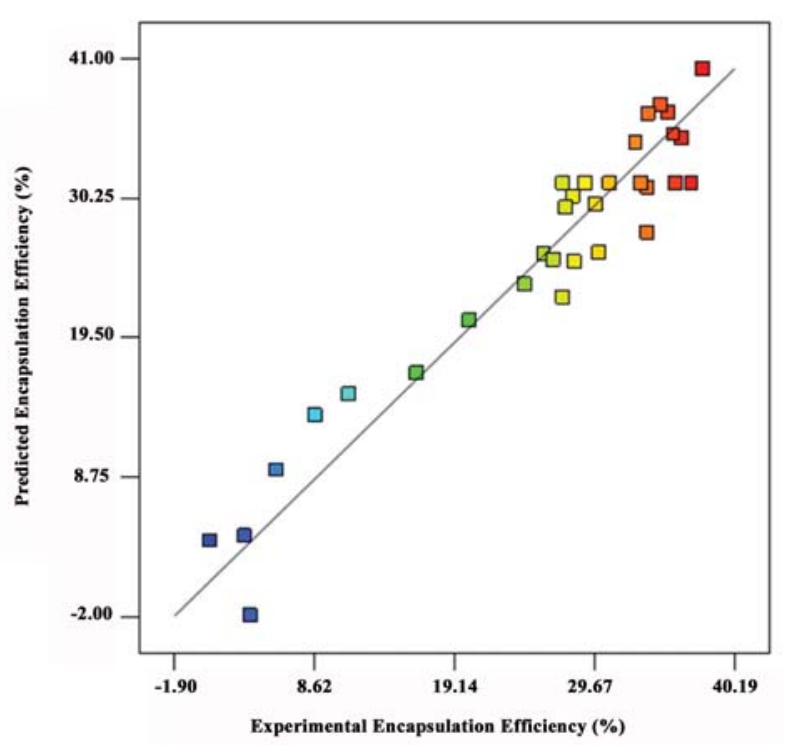

Figure 2. Predicted versus actual encapsulation efficiency (\%) of the eptifibatide into RMNL obtained from the model simulation and experiments, respectively. Points are the actual amount of DEE that approximately fits with the model due to high amount of R2 (0.9197) 


\subsection{Experimental Verification}

Suitability of the obtained optimum condition was validated by running an experiment (Table 4). The results of the experiment are quite close to the predicted values at a $95 \%$ of the confidence. The experimental result obtained for DEE was $37.49 \pm 5 \%$ which is within the estimated confidence interval range (35.1641.55).

\subsection{Characterization of the RMNL}

TEM and dynamic light scattering (DLS) analysis were used for characterizing the prepared RMNL. The results obtained from TEM analysis revealed that the size of RMNL is approximately in range of 60-90 nm (Figure 3). As well, DLS results showed that size of RMNL did not change at different pHs (Table 5). The neutral surface charge of RMNL, as shown by measurement of the $\zeta$ potential, did not significantly change at different $\mathrm{pH}$ (Table 5).

\subsection{The effect of SLS on Eptifibatide Encapsulation Efficiency and Drug Release}

The results show that SLS effect on encapsulation efficiency of eptifibatide was not significant (Figure 4A). In addition, eptifibatide release from RMNL and UNL in SBF during $24 \mathrm{~h}$ was 18 and $10 \%$ of the incorporated drug respectively (Figure 4B), whereas, drug release from RMNL and UNL was significantly enhanced in SBF containing SLS (40 and $27 \%$ of the incorporated drug during $24 \mathrm{~h}$ respectively, (Figure $4 C)$.

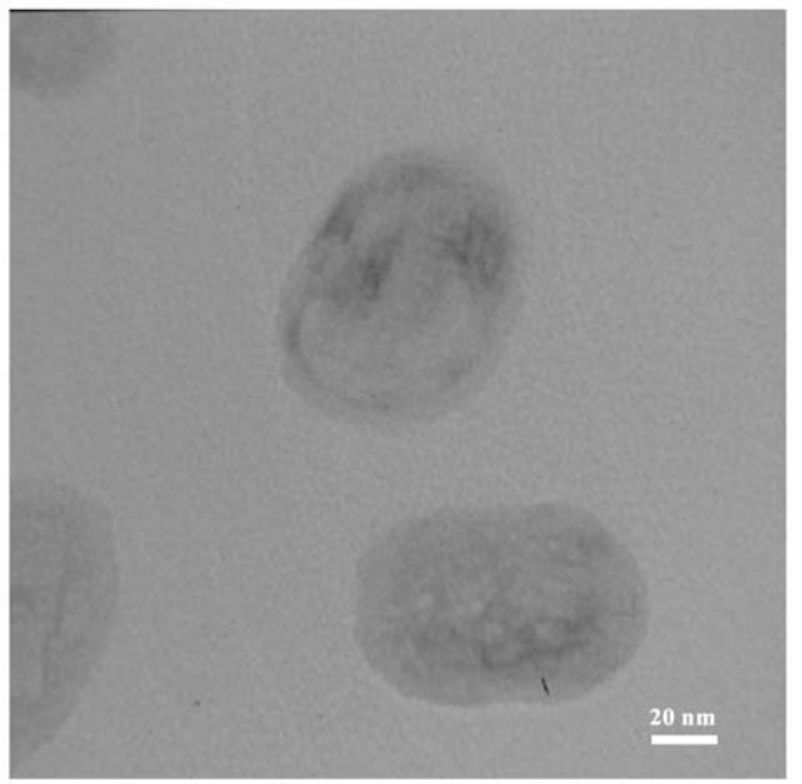

Figure 3. TEM images of the phospholipid-based bilayer vesicles containing eptifibatide prepared by freeze/thawing method
Table 5. Size and $\zeta$ potential measurement of RMNL at different $\mathrm{pH}$

\begin{tabular}{lcc}
\hline $\mathrm{pH}$ & Zeta $(\mathrm{mV})$ & Size $(\mathrm{nm})$ \\
\hline 5.5 & -2.96 & 92.11 \\
7.4 & -1.44 & 98.21 \\
8.5 & +0.639 & 95.08 \\
\hline
\end{tabular}

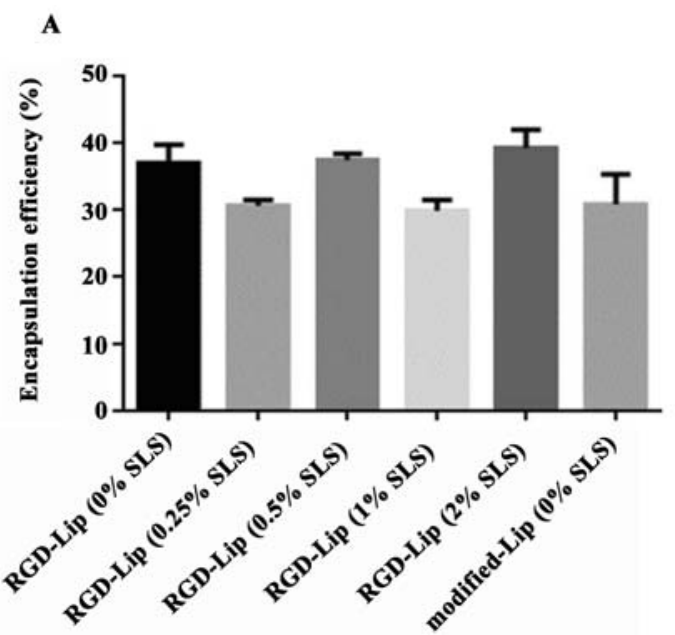

B

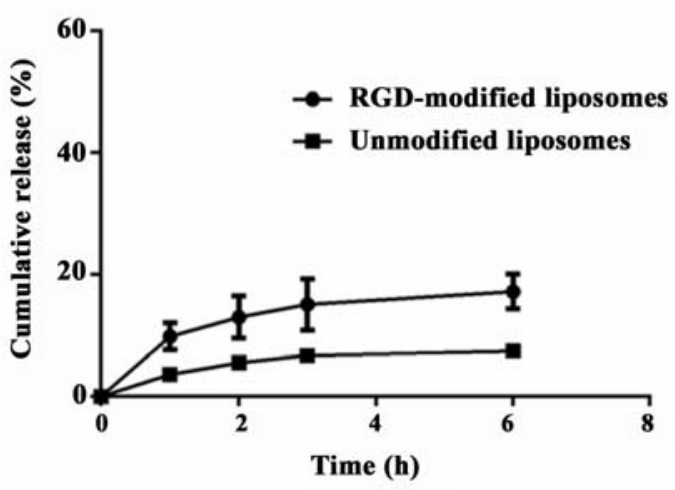

C

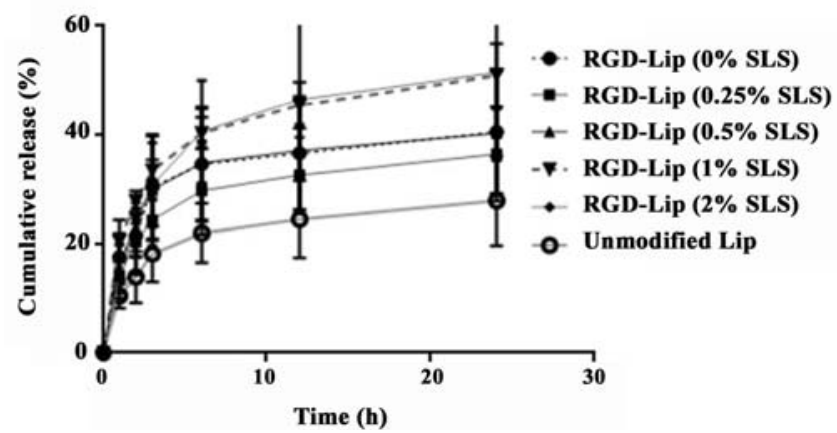

Figure 4. A: The effect of different concentration of SLS (0.25, $0.5,1$, and $2 \%$ SLS incorporated DSPC) on DEE and cumulative drug release profile of the eptifibatide-loaded liposomes at $37^{\circ} \mathrm{C}$, B: only SBF, and C: SBF containing $0.5 \%$ SLS 
In addition, SLS incorporated into RMNL ( 1 and $2 \%$ ol SLS per moL of DSPC) led to a larger drug release (up to $52 \%$ ) compared to UNL and RMNL without SLS (Figure 4C).

\section{Discussion}

Targeted drug delivery to the site of thrombus using fibrin specific or activated platelets specific ligands has recently become increasingly attractive $(19,20)$. Different peptides have been identified for targeting the various constituents of thrombus such as activated platelets (21). There are reports that have demonstrated the high affinity of the RGD peptide for GPIIb/IIIa receptor on surface of the activated platelets $(10,11$, 22 ). In the present study, a RGD motif was used as a ligand to modify the liposome surface for targeting the activated platelet cells.

The optimization of eptifibatide encapsulation into RMNL by RSM has demonstrated a significant correlation between independent variables. Statistical analysis through application of ANOVA has further certified the suitability of the reduced Quadratic model for determination of the relationship between the variables and encapsulation efficiency (Table 4). All of the independent factors -except dipalmitoyl- GRGDSPA peptide concentration have significantly positive effect on encapsulation efficiency.

Increasing the number of freeze/thawing cycles results in the enhancement of encapsulation efficiency due to an increase in the permeability of the lipid bilayer when liposome suspensions are immersed in the liquid nitrogen. In the liquid nitrogen, ice crystals are formed across lipid bilayers $(23,24)$, and cause lipid fragmentation during the freezing process. During the thawing step, water channels and pores are formed as a result of ice crystals melting. Under these conditions, drug molecules are passively transferred into the inner volume of liposomes. Furthermore, according to cryopreservation experiments, ice crystals cause leakage of the incorporated drug from liposomes (25-27). With heating the liposomes above the lipid phase transition temperature (Tc), fragmented phospholipids restore their structure and rebuild liposomes. Eptifibatide concentration also has a significant influence on the encapsulation efficiency. At higher eptifibatide concentration, the encapsulation efficiency is enhanced due to an increased entrapment of water containing hydrophilic drug. Encapsulation efficiency for hydrophilic drugs is proportional to the entrapped water volume into the inner volume of liposomes (28). Encapsulation efficiency significantly increases by increasing the concentration of the phospholipids (DSPC) due to the enhanced number of liposomes and consequently, an increased total internal volume of the liposomes (27). The significant effect of the cholesterol on encapsulation efficiency may be related to the interaction between cholesterol and eptifibatide.

The results of drug release have confirmed the high stability of the prepared RMNL causes low drug release, which is due to acyl chain length of phospholipid (DSPC) incorporated into RMNLs. This, in turn, is directly proportional to the high transition temperature $(\mathrm{Tc})$ of DSPC $\left(\mathrm{Tc}=55^{\circ} \mathrm{C}\right)(29,30)$. The increase in the drug release in the presence of SLS is due to the partial destabilization of RMNLs (31). Additionally, the high stability of the prepared liposomes by DSPC is suitable for in vivo application. The Tc is above body temperature; therefore reduces liposomal permeability and rate of leakage in the plasma at $37^{\circ} \mathrm{C}(29,32)$.

At the end of this study, the critical parameters for achieving an optimal encapsulation of eptifibatide into RMNL were identified; an attractive candidate for further in vitro and in vivo studies. Excepting dipalmitoylGRGDSPA peptide concentration, all other factors had significantly positive effect on encapsulation efficiency. The maximum DEE (38\%) was obtained with 7 cycles of freeze and thaw, $3.65 \mu$ mol eptifibatide, 7 $\mathrm{mM}$ DSPC, $3 \mathrm{mM}$ cholesterol and $1 \mathrm{mM}$ dipalmitoylGRGDSPA peptide, respectively. Our findings has provided essential information for the development of RMNL formulations in order to protect and enhance antithrombotic drugs efficacy.

\section{Funding/Support}

This work was financially supported by Tarbiat Modares University, Shahid Beheshti University of Medical Sciences, and Iran National Science Foundation (INSF) under grant No. 92013854.

\section{References}

1. Sanz J, Fayad ZA. Imaging of atherosclerotic cardiovascular disease. Nature 2008;451:953-957. DOI: 10.1038/nature06 803

2. Tcheng JE, O'Shea JC. Eptifibatide: a potent inhibitor of the platelet receptor integrin, glycoprotein IIb/IIIa. Expert Opin Investig Drugs. 1999;8(11):1893-1905. DOI: 10.1517/13543 784.8.11.1893

3. Addeo R, Faiola V, Guarrasi R, Montella L, Vincenzi B, Capasso E, et al. Liposomal pegylated doxorubicin plus vinorelbine combination as first-line chemotherapy for metastatic breast cancer in elderly women $>$ or $=65$ years of age. Cancer Chemother Pharmacol. 2008;62(2):285-292. DOI: 10.1007/s00280-007-0605-6

4. Torchilin VP. Targeting of drugs and drug carriers within the 
cardiovascular system. Adv Drug Deliv Rev. 1995;17:75-101. DOI: 10.1016/0169-409X(95)00042-6

5. Schiener M, Hossann M, Viola JR, Ortega-Gomez A, Weber $\mathrm{C}$, Lauber $\mathrm{K}$, et al. Nanomedicine-based strategies for treatment of atherosclerosis. Trends Mol Med. 2014;20(5):271281. DOI: 10.1016/j.molmed.2013.12.001

6. Haller CA, Cui W, Wen J, Robson SC, Chaikof EL. Reconstitution of CD39 in liposomes amplifies nucleoside triphosphate diphosphohydrolase activity and restores thromboregulatory properties. J Vasc Surg. 2006;43(4):816-823. DOI: $10.1016 /$ j.jvs.2005.11.057

7. Akbarzadeh A, Rezaei-Sadabady R, Davaran S, Joo SW, Zarghami N, Hanifehpour Y, et al. Liposome: classification, preparation, and applications. Nanoscale Res Lett. 2013;8(1):102. DOI: 10.1186/1556-276X-8-102

8. Srinivasan R, Marchant RE, Gupta AS. In vitro and in vivo platelet targeting by cyclic RGD-modified liposomes. $J$ Biomed Mater Res A. 2010;93(3):1004-1015. DOI: 10.1002/ jbm.a.32549

9. Vaidya B, Nayak MK, Dash D, Agrawal GP, Vyas SP. Development and characterization of site specific target sensitive liposomes for the delivery of thrombolytic agents. Int $J$ Pharm. 2011;403:254-261. DOI: 10.1016/j.ijpharm.2010.10. 028

10. Huang G, Zhou Z, Srinivasan R, Penn MS, Kottke-Marchant K, Marchant RE, et al. Affinity Manipulation of Surface-conjugated RGD-peptide to Modulate Binding of Liposomes to Activated Platelets. Biomaterials 2008;29(11):1676-1685. DOI: 10.1016/j.biomaterials.2007.12.015

11. Lestini BJ, Sagnella SM, Xu Z, Shive MS, Richter NJ, Jayaseharan J, et al. Surface modification of liposomes for selective cell targeting in cardiovascular drug delivery. $J$ Controlled Release. 2002;78:235-247. DOI: 10.1016/S01683659(01)00505-3

12. Goodman SL, Cooper SL, Albrecht RM. Integrin receptors and platelet adhesion to synthetic surfaces. J Biomed Mater Res. 1993;27(5):683-695. DOI: 10.1002/jbm.820270516

13. Fields GB, Noble RL. Solid phase peptide synthesis utilizing 9-fluorenylmethoxycarbonyl amino acids. Int J Pept Protein Res. 1990;35(3):161-214. DOI: 10.1111/j.1399-3011.1990. tb00939.x

14. Colletier JP, Chaize B, Winterhalter M, Fournier D. Protein encapsulation in liposomes: efficiency depends on interactions between protein and phospholipid bilayer. $B M C$ Biotechnol. 2002;2:9. DOI: 10.1186/1472-6750-2-9

15. Thiagarajan P, Kelly KL. Exposure of binding sites for vitronectin on platelets following stimulation. J Biol Chem. 1988;263(6):3035-3038.

16. Schachter DM, Kohn J. A synthetic polymer matrix for the delayed or pulsatile release of water-soluble peptides. $J$ Control Release. 2002;78(1):143-153. DOI: 10.1016/S01683659(01)00487-4

17. Kouchakzadeh H, Shojaosadati SA, Maghsoudi A, Vasheghani Farahani E. Optimization of PEGylation conditions for BSA nanoparticles using response surface methodology. AAPS PharmSciTech. 2010;11:1206-1211. DOI: 10.1208/s12249010-9487-8

18. Rocky-Salimi K, Hamidi-Esfahani Z, Abbasi S. Statistical optimization of arachidonic acid production by Mortierella alpina CBS 754.68 in submerged fermentation. Iran $J$ Biotech. 2011;9(2):87-93.

19. Erik Westein UF, Christoph E. Hagemeyer and Karlheinz Peter. Destination Known: Targeted Drug Delivery in Atherosclerosis and Thrombosis. Drug Dev Res. 2013;74:460-471. DOI: 10.1002/ddr.21103

20. Zhang J, Ma G, Lv Z, Zhou Y, Wen C, Wu Y, et al. Targeted thrombolysis strategies for neuroprotective effect. Neural Regen Res. 2014;9(13):1316-1322. DOI: 10.4103/16735374.137580

21. Vyas SP. Vaidya B. Targeted delivery of thrombolytic agents: role of integrin receptors. Expert Opin Drug Deliv. 2009;6(5):499-508. DOI: 10.1517/17425240902878002

22. Gupta AS, Huang G, Lestini BJ, Sagnella S, Kottke-Marchant $\mathrm{K}$, Marchant RE. RGD-modified liposomes targeted to activated platelets as a potential vascular drug delivery system. Thromb Haemost. 2005;93(1):106-114. DOI: 10.1160/TH0406-0340

23. Castile JD, Taylor KM. Factors affecting the size distribution of liposomes produced by freeze-thaw extrusion. Int J Pharm. 1999;188(1):87-95. DOI: 10.1016/S0378-5173(99)00207-0

24. Xu X, Costa A, Burgess DJ. Protein encapsulation in unilamellar liposomes: high encapsulation efficiency and a novel technique to assess lipid-protein interaction. Pharm Res. 2012;29(7):1919-1931. DOI: 10.1007/s11095-012-0720-x

25. Holovati JL, Gyongyossy-Issa MI, Acker JP. Effects of trehalose-loaded liposomes on red blood cell response to freezing and post-thaw membrane quality. Cryobiology 2009;58(1):75-83. DOI: 10.1016/j.cryobiol.2008.11.002

26. Siow LF, Rades T, Lim MH. Characterizing the freezing behavior of liposomes as a tool to understand the cryopreservation procedures. Cryobiology. 2007;55:210-221. DOI: 10.1016/j.cryobiol.2007.08.002

27. Ducat E BM, Lecomte F, Evrard B, Piel G. The experimental design as practical approach to develop and optimize a formulation of peptide-loaded liposomes. AAPS PharmSciTech. 2010;11(2):966-975. DOI: 10.1208/s12249-010-9463-3

28. Kulkarni SB, Betageri GV, Singh M. Factors affecting microencapsulation of drugs in liposomes. J Microencapsul. 1995;12(3):229-246. DOI: 10.3109/02652049509010292

29. Anderson M, Omri A. The effect of different lipid components on the in vitro stability and release kinetics of liposome formulations. Drug Deliv. 2004;11(1):33-39. DOI: 10.1080/ 10717540490265243

30. Alavizadeh SH, Badiee A, Golmohammadzadeh S, Jaafari MR. The influence of phospholipid on the physicochemical properties and anti-tumor efficacy of liposomes encapsulating cisplatin in mice bearing C26 colon carcinoma. Int J Pharm. 2014;473(1-2):326-333. DOI: 10.1016/j.ijpharm.2014.07.020

31. Duangjit S, Pamornpathomkul B, Opanasopit P, Rojanarata T, Obata Y, Takayama K, et al. Role of the charge, carbon chain length, and content of surfactant on the skin penetration of meloxicam-loaded liposomes. Int $J$ Nanomedicine. 2014;9:2005-17. DOI: 10.2147/IJN.S60674

32. Senior J. Fate and behavior of liposomes in vivo: a review of controlling factors. Crit Rev Ther Drug Carrier Syst. 1986;3(2):123-193. 